\section{Themenkomplex VII: Chirurgische Therapie}

K. H. Fuchs, W. Fischbach, J. Labenz, C. Zornig, H. Feussner

\section{Diagnostik \\ Konsens}

Zur präoperativen Diagnostik werden Endoskopie, ÖsophaguspH-Metrie und Manometrie zur optimalen Patientenselektion empfohlen (C).

\section{Kommentar}

Die chirurgisch relevanten pathophysiologischen Faktoren sind die Inkompetenz des unteren ösophagealen Sphinkters sowie eine erhöhte Anzahl transienter Sphinkterrelaxationen [1-6]. Für die Erwägung einer chirurgischen Therapie bei Patienten mit thorakalen und Oberbauchsymptomen ist es wichtig, dass eine gastroösophageale Refluxkrankheit sicher gemäß den allgemein gültigen Definitionen vorliegt. Eine chirurgische Therapie kommt prinzipiell in Betracht bei Vorliegen von Symptomen und einem pathologischen Befund in Endoskopie (Refluxösophagitis/Barrett) und/oder der 24-h-pH-Metrie (Evidenzgrad: C) [7-10]. Endoskopie und Ösophagus-pH-Metrie dienen der Objektivierung der Diagnose, die Manometrie dient dem Ausschluss anderer ösophagealer Erkrankungen, bei denen eine Antirefluxoperation eine Kontraindikation wäre. Die Manometrie ist für die Auswahl des chirurgischen Verfahrens nicht notwen$\operatorname{dig}[7,8,10-14]$.

\section{Indikationskriterien für eine Antirefluxoperation Konsens}

Voraussetzung einer chirurgischen Therapie ist eine langfristige Behandlungsnotwendigkeit. Unstrittige Indikationskriterien sind eine nachgewiesene Medikamentenunverträglichkeit, intolerable Restbeschwerden (z.B. aufgrund von Regurgitation und Aspiration) trotz adäquater medikamentöser Therapie. Eine relative Indikation besteht bei Patientenwunsch (C).

\section{Kommentar}

Für die Indikationsstellung zur Antirefluxoperation gilt die Prämisse, dass eine Notwendigkeit zu einer langfristigen Behandlung des Refluxes (in der Regel länger als 12 Monate) gegeben sein sollte. Bei der Unverträglichkeit auf Medikamente sollte auf die Möglichkeit eines Präparatewechsels unbedingt hingewiesen werden. Großvoluminöser Reflux ist medikamentös häufig schlecht zu beeinflussen. Die Ablehnung des Patienten gegenüber einer medikamentösen Langzeittherapie trotz nachgewiesener Wirksamkeit und guter Verträglichkeit von Protonpumpeninhibitoren muss eine Einzelentscheidung sein [7-9, 10, 15-24].

Prädiktive Faktoren für ein gutes postoperatives Ergebnis sind gutes symptomatisches Ansprechen auf Protonenpumpenblocker, eine nachgewiesene pathologische Säureexposition der Speiseröhre durch pH-Metrie und die Präsenz typischer Refluxsymptome [10].

\section{Kontraindikationen gegen die Antirefluxchirurgie} Konsens

Neben einem reduzierten Allgemeinzustand des Patienten gelten prinzipiell alle allgemeinen und chirurgischen Kontraindikationen. Bei psychisch auffälligen Patienten sollte die Operationsindikation besonders kritisch geprüft werden (C).

\section{Offene oder laparoskopische Antirefluxoperation Konsens}

Die laparoskopische Fundoplicatio sollte heute dem konventionellen Vorgehen vorgezogen werden $(A)$.

\section{Kommentar}

Es ist durch mehrere randomisierte Studien belegt, dass die Ergebnisse der laparoskopischen Antirefluxoperation denen mittels offener Technik überlegen sind. Insbesondere im Hinblick auf die Parameter postoperative Befindlichkeit, Lungenfunktion, Immunfunktion, postoperativer Schmerz, frühe Mobilisierung und Aktivitätsaufnahme sowie Reduktion der Milzläsionen intraoperativ hat die laparoskopische Operation signifikante Vorteile [25-30].

\section{Kontrolle der Refluxsymptome und Ergebnisse durch Antirefluxchirurgie \\ Konsens}

Die Antirefluxchirurgie ist der medikamentösen Therapie hinsichtlich Beseitigung der Refluxbeschwerden gleichwertig innerhalb eines Zeitraumes von 5 - 10 Jahren (A). Überlegenheit einer Behandlungsmethode hinsichtlich des Langzeitverlaufs als auch der Kosten ist weder für eine medikamentöse Therapie noch für die Antirefluxoperation gesichert (C). Die operationsbedingte Letalität der laparoskopischen Antirefluxoperation liegt zwischen $0 \%$ und $0,6 \%$.

\section{Kommentar}

Folgende Kriterien sind für die Erfolgsbeurteilung einer medikamentösen und chirurgischen Therapie relevant: 1 . Symptomkontrolle, 2. Verbesserung der Lebensqualität, 3. Abheilung der Ösophagitis und 4. therapiebedingte Morbidität und Mortalität.

In der bisher einzigen Vergleichsstudie Antirefluxchirurgie versus Protonenpumpenblocker waren beide Verfahren gleich wirksam, wenn die individuelle Dosisanpassung des Protonenpumpenblockers zugelassen wurde. Aus den vorliegenden Ergebnissen weiterer prospektiver Studien liegt die Erfolgsrate der offenen Chirurgie nach 5 Jahren zwischen 83-95\%, nach 5-10 Jahren zwischen $66-96 \%$ und nach mehr als 10 Jahren Nachsorgezeit zwischen 56-85\%. Nach Anwendung der laparoskopischen Technik liegen die Erfolgsraten mit 85-95\% nach 5 Jahren vergleichbar hoch. In den wenigen verfügbaren Studien mit Ergebnissen bei mehr als 5 Jahren Nachsorgezeit nach laparoskopischer Operation wird eine Erfolgsrate von 85\% angegeben [7, 8, 20, 21 - 30]. Ergebnisse aus nicht spezialisierten Zentren oder aus kleineren Abteilungen zeigen aber deutlich schlechtere Ergebnisse [31, 32].

Anhand von Studienergebnissen aus spezialisierten Zentren ist die Letalität niedrig und liegt unter 0,5\%. Für die Prognose der gastroösophagealen Refluxkrankheit quo ad vitam ist eine Überlegenheit der medikamentösen oder operativen Therapie nicht 
belegt [7, 8, 28 - 30, 33, 34]. Dies gilt auch für Patienten mit einem Barrett-Ösophagus.

Die direkten und indirekten Kosten der operativen und konservativen Therapie der gastroösophagealen Refluxkrankheit werden maßgeblich vom jeweiligen Gesundheitssystem bestimmt. Vergleichende Kosten-Nutzen-Analysen aus anderen Ländern sind daher nicht uneingeschränkt auf deutsche Verhältnisse zu übertragen [35-44].

\section{Vorgehen bei Versagen der Antirefluxoperation Konsens}

Versager der Antirefluxoperation sind operationstechnisch bedingt (Weite bzw. Länge der Manschette) oder entstehen durch Migration der Manschette (B). Klinisch imponieren sie als Refluxrezidiv, Dysphagie und/oder Gas-Bloat-Symptome.

Refluxrezidive sollten medikamentös behandelt werden. Weitere Diagnostik (Endoskopie, Radiographie) ist indiziert bei klinisch ausgeprägter postoperativer Symptomatik (C). Schwere Dysphagie oder andere Symptom-Kombinationen bedürfen weiterer Abklärung und gegebenenfalls der Revision.

\section{Kommentar}

Bei Persistenz oder Rezidiv der Refluxsymptome sollte eine medikamentöse Therapie begonnen werden. In der Folgezeit gelten für diese Patienten dann Kriterien ähnlich wie bei einer primären Refluxkrankheit bezüglich einer erneuten Operationsindikation. Nur bei klinisch relevanter postoperativer Morbidität, insbesondere persistierender Dysphagie, sollte man rasch auf eine diagnostische Klärung drängen und ggf. eine erneute Operation erwägen [7, 8, 14, 45, 46].

Mechanismen des Versagens sind transdiaphragmale Migration der Fundoplicatio als häufigste Ursache, der sog. slipped Nissen, auch Teleskop-Phänomen genannt, oder - davon manchmal relativ schlecht zu trennen - die schlecht platzierte Fundoplicatio bzw. die zu tief platzierte Fundoplicatio, die verdrehte Anlage der Manschette sowie eine zu eng angelegte oder zu lang angelegte Manschette. Diese führen zum Refluxrezidiv und/oder zur persistierenden Schluckstörung. Hinzu kommt die Auflösung der Manschette durch das Durchziehen des Fadenmaterials durch die Magenwand bzw. die Ösophaguswand oder gar in seltenen Fällen die Verwendung von resorbierbarem Nahtmaterial [7, 8, $45,46]$. Falsche Indikationsstellung kann eine erhöhte postoperative Morbidität begünstigen.

\section{Literatur}

${ }^{1}$ DeMeester TR, Johnson LS, Joseph GJ et al. Patterns of gastroesophageal reflux in health and disease. Ann Surg 1976; 184: 459-470

2 Fuchs KH, Freys SM, Heimbucher J et al. Pathophysiologic spectrum in patients with gastroesophageal reflux disease in a surgical GI function laboratory. Diseases of the Esophagus 1995; 8: 211 - 217

${ }^{3}$ Dent J, Holloway RH, Toouli J et al. Mechanisms of lower oesophageal sphincter incompetence in patients with symptomatic gastro-oesophageal reflux. Gut 1988; 29: 1020-1028

${ }^{4}$ Mittal RK, Holloway RH, Penagini R et al. Transient lower esophageal sphincter relaxations. Gastroenterology 1995; 109: 601 -610

${ }^{5}$ Zaninotto G, DeMeester TR, Schwizer W et al. The lower esophageal sphincter in health and disease. Am J Surg 1988; 155: 104 - 111
${ }^{6}$ Johnsson F, Holloway RH, Ireland AC et al. Effect of fundoplication on transient lower oesophageal sphincter relaxation and gas reflux. $\mathrm{Br} \mathrm{J}$ Surg 1997; 84: 686-689

${ }^{7}$ Eypasch E, Neugebauer E, Fischer F et al. Laparoscopic antireflux surgery for gastroesophageal reflux disease (GERD). Results of a consensus development conference. Surg Endoscopy 1997; 11: 413-426

${ }^{8}$ Fuchs $\mathrm{KH}$, Feussner $\mathrm{H}$, Bonavina L et al. Current status and trends in laparoscopic antireflux surgery: results of a consensus meeting. Endoscopy 1997; 29: $298-308$

${ }^{9}$ Dent J, Brun J, Fendrick AM et al. Geneval Workshop Group: An evidence-based appraisal of reflux disease management. Gut 1999; 44: S1-S16

10 Campos GM, Peters JH, DeMeester TR et al. Multivariate analysis of factors predicting outcome after laparoscopic Nissen fundoplication. J Gastrointest Surg 1999; 3 (3): 292 - 300

${ }^{11}$ Rydberg L, Ruth M, Abrahamsson $\mathrm{H}$ et al. Tailoring antireflux surgery: a randomized clinical trial. World J Surg 1999; 23: 612 - 618

${ }^{12}$ Fibbe C, Layer P, Keller J et al. Esophageal motility in reflux disease before and after fundoplication: a prospective, randomized, clinical and manometric study. Gastroenterology 2001; 121: 5-14

${ }^{13}$ DeMeester TR, Johnson LF, Kent AH. Evaluation of current operations for the prevention of gastroesophageal reflux. Ann Surg 1974; 180: $511-525$

${ }^{14}$ Dallemagne B, Weerts JM, Jehaes C et al. Causes of failures of laparoscopic antireflux operations. Surg Endosc 1996; 10: 305-310

${ }^{15}$ Larrain A, Carrasco E, Galleguillos F et al. Medical and surgical treatment of nonallergic asthma associated with gastroesophageal reflux. Chest 1991; 99: 1330-1335

${ }^{16}$ Allen CJ, Anvari M. Gastro-oesophageal reflux related cough and its response to laparoscopic fundoplication. Thorax 1998; 53: 963-968

17 So JB, Zeitels SM, Rattner DW. Outcomes of atypical symptoms attributed to gastroesophageal reflux treated by laparoscopic fundoplication. Surgery 1998; 124 (1): 28-32

${ }^{18}$ Kahrilas PJ. Surgical therapy for reflux disease. JAMA 2001; 285: 2376

${ }^{19}$ Grande L, Toledo-Pimentel V, Manterola C et al. Value of Nissen fundoplication in patients with gastro-oesophageal reflux judged by longterm symptom control. Br J Surg 1994; 81 (4): 548-50

${ }^{20}$ Hinder RA, Filipi CJ, Wetscher G et al. Laparoscopic Nissen fundoplication is an effective treatment for gastroesophageal reflux disease. Ann Surg 1994; 220: $472-483$

${ }^{21}$ Allgood PC, Bachmann M. Medical or surgical treatment for chronic gastro-oesophageal reflux? A systematic review of published evidence of effectiveness. Eur J Surg 2000; 166: 713 - 721

${ }^{22}$ Lundell L, Miettinen P, Myrvold HE et al. Continued (5-year) followup of a randomized clinical study comparing antireflux surgery and omeprazole in gastroesophageal reflux disease. J Am Coll Surg 2001; 192 (2): 172 - 179; discussion 179-281

${ }^{23}$ Klinkenberg-Knol EC, Nelis F, Dent J et al. Long-term Omeprazole treatment in resistant gastroesophageal reflux disease: efficacy, safety, and influence on gastric mucosa. Gastroenterology 2000; 118: $661-669$

${ }^{24}$ Spechler SJ, Lee E, Ahnen D et al. Long-term outcome of medical and surgical therapies for gastroesophageal reflux disease. JAMA 2001; 285: $2331-2338$

${ }^{25}$ Laine S, Rantala A, Gullichsen R et al. Laparoscopic vs conventional Nissen fundoplication. A prospective randomized study. Surg Endosc 1997; 11: $441-444$

${ }^{26}$ Bais JE, Bartelsman JF, Bonjer HJ et al. Laparoscopic or conventional Nissen fundoplication for gastroesophageal reflux disease: randomized clinical trial. Lancet 2000; 355: 170-174

${ }^{27}$ Nilsson G, Larsson S, Johnsson F. Randomized clinical trial of laparoscopic versus open fundoplication: blind evaluation of recovery and discharge period. Br J Surg 2000; 87: 873-878

28 Zaninotto G, Molena D, Ancona E Study Group for the Laparoscopic Treatment of Gastroesophageal Reflux Disease of the Italian Society of Endoscopic Surgery. A prospective multicenter study on laparoscopic treatment of gastroesophageal reflux disease in Italy. Surg Endosc 2000; $14: 282-288$

${ }^{29}$ Hunter JG, Smith CD, Branum GD et al. Laparoscopic fundoplication failures. Ann Surg 1999; 230: 595-606

${ }^{30}$ Perdikis G, Hinder RA, Lund RJ et al. Laparsocopic Nissen fundoplication: Where do we stand? Surg Laparoscopy \& Endoscopy 1997; 7: $17-21$ 
${ }^{31}$ Rantanen TK, Halme TV, Loustarinen ME et al. The long term results of open antireflux surgery in a community-based health care center. Am J Gastroenterol 1999; 94: 1777-1781

32 Vakil N, Shaw M, Kirby R. Clinical effectiveness of laparoscopic fundoplication in a U.S. community. Am J Med 2003; 114: 1 - 5

${ }^{33}$ Rantanen TK, Salo JA. Gastroesophageal reflux disease as a cause of death: analysis of fatal cases under conservative treatment. Scand J Gastreoenterol 1999; 3: 229-233

34 Rantanen TK, Salo JA, Sipponen JT. Fatal and life-threatening complications in antireflux surgery: analysis of 5502 operations. Br J Surg 1999; 86: $1573-1577$

35 Trus TL, Laycock WS, Waring JP et al. Improvement in quality of life measures after laparoscopic antireflux surgery. Ann Surg 1999; 229 (3): $331-336$

36 Sandbu R, Hallgren T. The economics of laparoscopic antireflux operations compared with open surgery. Eur J Surg 2000; Suppl 585: 37 - 39

${ }^{37}$ Granderath FA, Kamolz T, Schweiger UM et al. Lebensqualität und subjektive Beurteilung der Ergebnisqualität 3 Jahre nach laparoskopischer Antirefluxchirurgie. Der Chirurg 2000; 71: 950-954

38 Kamolz T, Wykypiel H, Bammer T et al. Lebensqualität nach laparoskopischer Antirefluxchirurgie - Nissen-Fundoplicatio. Der Chirurg 1998; 69: 947 -950
${ }^{39}$ Lönroth H. Efficacy of, and quality of life after antireflux surgery. Eur J Surg 2000; 166: $34-36$

40 Jönsson B, Zethraeus N. Costs and benefits of laparoscopic surgery - a review of the literature. Eur J Surg 2000; Suppl 585: 48 - 56

${ }^{41}$ Heudebert GR, Marks R, Wilcox CM et al. Choice of long-term strategy for the management of patients with severe esophagitis: a cost-utility analysis. Gastroenterology 1997; 112: 1078-1086

42 Van Den Boom G, Go PM, Hameeteman W et al. Cost effectiveness of medical versus surgical treatment in patients with severe or refractory gastroesophageal reflux disease in the Netherlands. Scand J Gastroenterol 1996; 31 (1): $1-9$

${ }^{43}$ Fuchs KH, Tigges H, Heimbucher J et al. Laparoskopische Eingriffe bei gastroösophagealer Refluxkrankheit - eine Nutzen-Kosten-Analyse. Zentralbl Chir 1997; 122: $1072-1077$

${ }^{44}$ Myrvold HE, Lundell L, Miettinen P et al. The cost of long term therapy for gastro-oesophageal reflux disease: a rondomised trial comparing omeprazole and open antireflux surgery. Gut 2001; 49: 488-494

${ }^{45}$ Stein HJ, Feussner H, Siewert JR. Failure of antireflux surgery: causes and management strategies. Am J Surg 1996; 171: 36-40

${ }^{46}$ Hunter JG, Smith CD, Branum GD et al. Laparoscopic fundoplication failures. Ann Surg 1999; 230: 595-606 
Dr. A. Lütke · Löhr-Center · Mainzer Straße 66 · 56068 Koblenz

Dr. Ahmed Madisch • Medizinische Klinik und Poliklinik · Universitätsklinikum Carl Gustav Carus · Technische Universität Dresden $\cdot$ Fetscherstraße $74 \cdot 01307$ Dresden

Prof. Dr. Peter Malfertheiner, PD Dr. Ulrich Peitz · Zentrum für Innere Medizin · Gastroenterologie und Hepatologie · Otto von Guerricke Universität · Leipziger Straße 44 · 39120 Magdeburg

PD Dr. Helmut Messmann · III. Medizinische Klinik · Klinikum Augsburg · Stenglinstraße 2 86156 Augsburg

Prof. Dr. G. Micklefield · Medizinische Klinik I · Evangelisches Krankenhaus Unna · Holbeinstraße 10 · 59423 Unna

Prof. Dr. Stefan Müller-Lissner · Abteilung Innere Medizin · ParkKlinik Weißensee · Lehrkrankenhaus der Charité · Schönstraße $80 \cdot 13086$ Berlin

Dr. Marianne Ortner · Klinik für Gastroenterologie · Centre Hospitalier Universitaire Vaudois - Rue de Bugnon 46 • CH-1011 Lausanne (Schweiz)
Prof. Dr. R. Porschen · Medizinische Klinik · Zentralkrankenhaus Bremen-Ost · Züricher Str. $40 \cdot 28325$ Bremen

Prof. Dr. K. Rasche · Kliniken St. Antonius - Akad. Lehrkrankenhaus der Heinrich-Heine-Universität Düsseldorf · Zentrum für Innere Medizin - Schwerpunkt Pneumologie - Carnaper Str. 48, 42283 Wuppertal

Prof. Dr. Wolfgang Rösch · Medizinische Klinik · Krankenhaus Nordwest · Steinbacher Hohl 2-26 · 60488 Frankfurt am Main

Prof. Dr. Wolfgang Schepp, PD Dr. Christian Pehl · 2. Medizinische Abteilung · Städtisches Krankenhaus München-Bogenhausen · Englschalkinger Straße $77 \cdot 81925$ München

Prof. Dr. M. Stolte · Pathologisches Institut - Klinikum Bayreuth • Preuschwitzer Str. $101 \cdot 95445$ Bayreuth

Prof. Dr. Martin Wienbeck - ehemals III. Medizinische Klinik • Klinikum Augsburg · Stenglinstraße 2 86156 Augsburg

Prof. Dr. C. Zornig · Chirurgische Klinik · Israelitisches Krankenhaus · Orchideenstieg $14 \cdot 22297$ Hamburg 